\title{
EFICIÊNCIA DO CONTROLE QUÍMICO DO ÁCARO ALARANJADO DO ABACAXIZEIRO, Dolichotetranychus floridanus (BANKS, 1900) (ACARI: TENUIPALPIDAE) EM PERNAMBUCO
}

\author{
Paulo Fernando Ferreira ${ }^{1}$ \\ Antônio Fernando de Souza Leão Veiga ${ }^{2}$
}

\section{RESUMO}

Para avaliar a eficiência dos inseticidas-acaricidas sistêmicos acefato, vamidothion, metamidophos e monocrotophos no controle do ácaro alaranjado do abacaxizeiro, Dolichotetranychus floridanus e o efeito sobre a produtividade, desenvolveu-se experimento na Estação Experimental de Itambé, da Empresa IPA, município de Itambé, Pernambuco, com a cultivar Pérola, utilizando-se mudas infestadas, oriundas de cultura geral. Testaram-se os seguintes tratamentos: Acefato (120g i.a./100L de água); Vamidothion (45g i.a./100L de água); Metamidophos (60g i.a./ 100L de água); Monocrotophos (60g i.a./100L de água) e a testemunha. Foram realizadas quatro aplicações com intervalos de 30 dias, a primeira efetuada aos 150 dias e as demais aos 180, 210 e 240 dias após o plantio (DAP). As aplicações foram realizadas imediatamente após a coleta das amostras de plantas para a contagem da população. Pesou-se a produção por parcela por ocasião da colheita e fizeram-se cinco contagens da população durante o ciclo da cultura. Na eficiência de controle do ácaro alaranjado; o Vamidothion foi o que mais se destacou, com 88,31\% de eficiência, até 270 dias após o plantio, entretanto não interferiu na produ-

1. Área de fruticultura do Programa de Fruticultura da Empresa Pernambucana de Pesquisa Agropecuária (IPA), Recife-PE, Brasil.

2. Área de Entomologia do Departamento de Biologia da Universidade Federal Rural de Pernambuco (UFRPE), Recife-PE. 
tividade. O Acefato apresentou-se como ineficiente.

Palavras-chave: abacaxi cv. Pérola, inseticidas-acaricidas sistêmicos, Dolichotetranychus floridanus, ácaro alaranjado do abacaxizeiro.

\section{ABSTRACT}

\section{EFFICIENCY OF THE CHEMICAL CONTROL OF ORANGE MITE OF PINEAPPLE, Dolichotetranych us floridanus (BANKS, 1900) (ACARI: TENUIPALPIDAE), IN PERNAMBUCO STATE, BRAZIL}

To evaluate the efficiency of the systemic insecticide-acaricide acephato, vamidothion, metamidophos and monocrotophos to control the orange mite of the pineaple, Dolichotetranychus floridanus and the effect on productivity, an experiment was developed at Experimental Station of Itambé, of Empresa IPA, district of Itambé, Pernambuco, Brazil, using infested seedlings of the cultivate Pérola from the general culture. The following treatments were tested: acephato $(120 \mathrm{~g}$ i.a. $/ 100 \mathrm{~L}$ of water); vamidothion $(45 \mathrm{~g}$ i.a. $/ 100 \mathrm{~L}$ of water); metamidophos $(60 \mathrm{~g}$ i.a. $/ 100 \mathrm{~L}$ of water); monocrotophos (60g i.a. $/ 100 \mathrm{~L}$ of water) and the control. Four applications were accomplished by intervals of 30 days, being the first one made 150 days after planting. The applications were accomplished immediately after the samples of plants were collected for population count. The production of the experimental unities was weighed at harvest and five counts of the population during the cycle of the culture were done. The results pointed out vamidothion as the best for orange mite control with $88,31 \%$ of efficiency, until 270 days after planting, but it didn't interfere on productivity. The acephato showed ineficiency on the mite.

Key words: pineapple cultivar Pérola, systemic insecticide-acaricide, Dolichotetranychus floridanus, orange mite of pineapple. 


\section{[NTRODUÇÃO}

O abacaxizeiro, Ananas comosus (L.) Merril, é uma planta tropizal originária de regiões de clima quente e seco ou de pluviosidade irrezular. É uma planta herbácea, monocotilidônea, semiperene, da família Bromeliaceae.

A sua importância é devida não só as qualidades do fruto, bastante apreciado em todo o mundo, mas principalmente pelo aspecto econômico e social da cultura, como atividade de uso intensivo de mão-de-obra rural, especialmente no Nordeste (Cunha et al., 1994).

O Brasil está colocado entre os três maiores países produtores de ábacaxi do mundo, após a Tailândia e Filipinas. As informações sobre a abacaxicultura mundial sinalizam, para o Brasil, possibilidades de conquistar novos mercados, como o norte-americano e o europeu (Chalfoun, 1998; Manica, 1999).

Há diversos problemas que impedem a expansão da produção do abacaxi no Brasil, especialmente no Nordeste, podendo-se salientar a ocorrência de pragas e doenças. No Estado de Pernambuco, tem-se destacado ha cultura do abacaxi a ocorrência do ácaro alaranjado, Dolichotetranychus floridanus (Banks, 1900). Arruda \& Flechtmann (1967) relataram pela primeira vez em Pernambuco a presença do ácaro alaranjado e que seu ataque, na base e bainhas das folhas das plantas, pode apresentar sintomas de murcha, quando em alta infestação. Segundo Reis (1981), o ácaro alaranjado, quando em ataques severos, causa redução de até $16 \%$ na produção.

Em relação ao controle químico do ácaro alaranjado, Sanches \& Zem (1978), desenvolveram, na Bahia, testes com diferentes inseticidasacaricidas em tratamento (aplicações) sobre mudas de abacaxizeiro cv. Pérola, utilizando sete produtos em três concentrações e dois tempos de imersão. Não encontraram diferenças significativas quanto ao tempo de imersão, mas constataram que os produtos Ometoato a 100 e $150 \mathrm{~mL} /$ $100 \mathrm{~L}$ d'água, Vamidothion a $225 \mathrm{~mL} / 100 \mathrm{~L}$ d'água e Ethion a $225 \mathrm{~mL} /$ $100 \mathrm{~L}$ d'água, foram os mais eficientes, com mortalidades inferiores a $100 \%$. 
Os autores constataram ainda ausência de ação ovicida, necessitando de controle pós-plantio. Na mesma época, Sanches (1978), testando vários produtos, recomendou o uso do Monocrotophos em desinfestação de mudas (pré-plantio) seguido de pulverização pós-plantio com Vamidothion a $0,6 \mathrm{~L}$ p.c./ha, iniciando após 90 dias do plantio, como eficiente no controle do ácaro alaranjado.

Arleu et al. (1981), no Espírito Santo, trabalhando com desinfestação das mudas por cinco minutos e pulverização em pós-plantio, verificaram que as combinações mais eficientes foram aquelas à base de Monocrotophos a $100 \mathrm{~mL} / 100$ litros de água, seguidas de pulverização no campo com Monocrotophos a 1,5 L/ha, Vamidothion a $0,6 \mathrm{~L} /$ ha e ainda Dicofol a 3,0 L/ha, iniciadas 218 dias após a desinfestação e repetidas a cada 30 dias, suspendendo-se 60 dias antes da colheita. Sampaio \& Myazaki (1982), em São Paulo, através de pulverização de mudas de abacaxizeiro acondicionadas em sacos de polietileno, infestadas pelo ácaro alaranjado, conseguiram mortalidade acima de $90,0 \%$, após $24 \mathrm{~h}$ da aplicação, com os seguintes produtos: Dimetoato a $0,15 \%$; Ethion a $0,1 \%$; Sumithoate a $0,1 \%$ e o Monocrotophos a $0,08 \%$, sem observar efeitos fitotóxicos.

O presente trabalho teve como objetivo testar a eficiência dos inseticidas-acaricidas sistêmicos: Acefato, Vamidothion, Metamidophos e Monocrotophos sobre o ácaro alaranjado e os efeitos sobre a produtividade do abacaxi.

\section{MATERIAL E MÉTODOS}

O experimento foi realizado na Estação Experimental de Itambé, da Empresa Pernambucana de Pesquisa Agropecuária (IPA), no município de Itambé-PE, em 1997/1998, com o cultivar Pérola. A área de cada parcela foi de $12,80 \mathrm{~m}^{2}$, constituída por quatro fileiras no total e duas linhas úteis com oito plantas cada, no espaçamento $1,0 \times 0,40 \mathrm{~m}$. Foram comparados cinco tratamentos; quatro inseticidas-acaricidas e 
Ima testemunha, com quatro blocos casualizados (Pimentel-Gomes, 1990) (Tabela 1).

Tabela 1. Inseticidas-acaricidas sistêmicos utilizados no controle do ácaro alaranjado Dolichotetranychus floridanus na cultura do abacaxi cv. Pérola. Itambé, PE, 1998.

\begin{tabular}{|c|c|c|c|c|}
\hline \multirow{2}{*}{ Tratamento } & \multirow{2}{*}{$\begin{array}{l}\text { Produto } \\
\text { Comercial }\end{array}$} & \multirow{2}{*}{$\begin{array}{l}\text { Concentração e } \\
\text { formulação }\end{array}$} & \multicolumn{2}{|c|}{ Doses (/100L d'água) } \\
\hline & & & p.c. & g.i.a. \\
\hline Acefato & Orthene & $750 \mathrm{BR} / \mathrm{PM}$ & $160,0 \mathrm{~g}$ & 120,0 \\
\hline Vamidothion & Kilval & $300 \mathrm{CE}$ & $150,0 \mathrm{~mL}$ & 45,0 \\
\hline Metamidophos & Hamidop & $600 \mathrm{SC}$ & $100,0 \mathrm{~mL}$ & 60,0 \\
\hline Monocrotophos & Azodrin & $400 \mathrm{SC}$ & $150,0 \mathrm{~mL}$ & 60,0 \\
\hline Testemunha & n......... & n......... & (n....... & .......... \\
\hline
\end{tabular}

Na adubação utilizaram-se $200 \mathrm{~g}$ por planta da mistura 10-5-16 de NPK, parcelada em quatro vezes, aos 30, 90, 180 e 270 dias do plantio. As mudas utilizadas foram do tipo filhote com 300 à $350 \mathrm{~g}$, obtido na orópria Estação Experimental, em cultura geral, infestada pelo ácaro llaranjado. Por ocasião da estiagem recorreu-se ao processo de rega. A ndução floral foi feita aos 12 meses após o plantio, depositando-se $2,0 \mathrm{~g}$ le carbureto de cálcio no interior da roseta foliar. A colheita e pesagem la produção foi realizada com 18 meses do plantio, na área útil de cada parcela.

Na proteção das parcelas contra o efeito de deriva utilizou-se uma portina protetora de material plástico, com 4,0m de comprimento, 3,5m le largura e 2,0m de altura, sustentada lateralmente por quatro caibros fom ponta em bisel. Foram realizadas quatro aplicações dos produtos nas parcelas, com intervalos de 30 dias, sendo a primeira aplicação efetuada os 150 dias e as demais aos 180, 210 e 240 dias após o plantio (DAP). As aplicações foram feitas por meio de pulverizador manual costal, a alto volume, com bico em jato cônico, dirigindo-se o jato da calda inseticida para as bainhas foliares. 
Na avaliação da infestação do ácaro alaranjado colheram-se duas plantas por parcela das fileiras de borda, totalizando oito plantas por tratamento e $40 \mathrm{em}$ todo o experimento. Houve coletas de amostras das plantas antes de cada aplicação dos produtos químicos. Foram observadas 10 folhas por planta e 80 por tratamento, no sentido da base para o ápice da roseta foliar obtendo-se uma média de ácaros vivos na planta. A contagem dos ácaros vivos foi realizada no laboratório de Entomologia da Universidade Federal Rural de Pernambuco (UFRPE). Para tal contou-se o número de ácaros presentes numa microárea de $1,0 \mathrm{~cm}^{2}$, de maior concentração de ácaros, em cada bainha foliar.

Os resultados obtidos (x) foram transformados em $\sqrt{x}$ e submetidos à análise de variância, e as médias comparadas pelo teste de Tukey ao nível de $5 \%$ de probabilidade. Os porcentuais de eficiência $(\% \mathrm{E})$ foram calculados pela fórmula de Abbott (1925): $\% \mathrm{E}=100(\mathrm{~T}-\mathrm{C}) / \mathrm{T}$, onde $\mathrm{T}=$ número de ácaros na testemunha e $\mathrm{C}=\mathrm{o}$ número de ácaros no tratamento.

\section{RESULTADOS E DISCUSSÃO}

Os dados obtidos referentes à eficiência dos inseticidas-acaricidas são apresentados na Tabela 2 . Observa-se que a infestação estava relativamente uniforme, antes de qualquer tratamento. Aos 30 dias após a primeira aplicação apenas os tratamentos com Vamidothion e Metamidophos diferiram significativamente da testemunha, mas não entre si. Já o tratamento com Acefato diferiu do tratamento com Metamidophos. Aos 30 dias após a segunda aplicação (DAP) o Vamidothion apresentou eficiência de $62,29 \%$ apesar de não ter havido diferença siginificativa em relação à testemunha e aos demais tratamentos. $\mathrm{O}$ Acefato foi o que apresentou a menor eficiência, com 19,89\%. Com 30 dias após a terceira aplicação o Vamidothion foi o único que diferiu significativamente da testemunha, mas não dos demais, apresentando a melhor eficiência, com $65,0 \%$ de redução da população. Aos 30 dias após a quarta aplicação o 


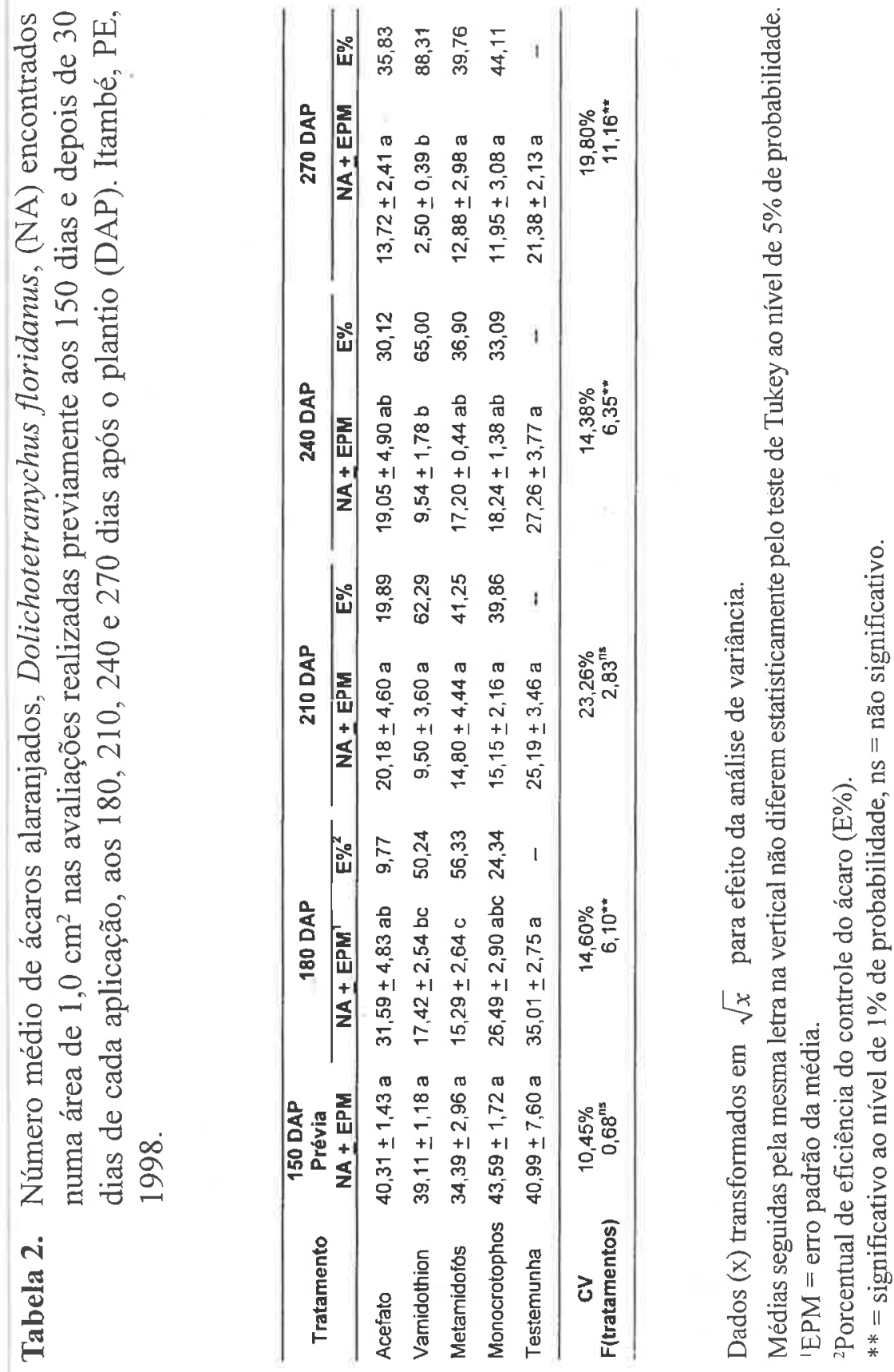


vamidothion apresentou uma eficiência de controle de 88,31\% diferindo significativamente dos demais tratamentos em relação ao número médio de ácaros, sendo o acefato aquele que apresentou menor eficiência, com 35,83\%.

De acordo com os dados da Tabela 3, não observaram-se diferenças entre os tratamentos para produtividade.

Tabela 3. Efeito de aplicações de inseticidas-acaricidas no controle do ácaro alaranjado, Dolichotetranychus floridanus, sobre a produtividade da cultura do abacaxi cv. Pérola. Itambé, PE, 1998.

\begin{tabular}{lc}
\hline Tratamentos & ${\text { Produtividade (t/ha) } \pm \text { EPM }^{2}}^{2}$ \\
Acefato & $21,08 \pm 2,39$ \\
Vamidothion & $20,83 \pm 2,35$ \\
Metamidophos & $21,32 \pm 3,50$ \\
Monocrotophos & $19,71 \pm 2,77$ \\
Testemunha & $19,50 \pm 2,38$ \\
\hline CV (\%) & 28,90 \\
F(tratamentos) & $0,08 \mathrm{NS}$ \\
\hline
\end{tabular}

'Valor do teste $\mathrm{F}$ para os tratamentos. NS = não significativo. ${ }^{2} \mathrm{EPM}=$ erro padrão da média.

Apesar de o Vamidothion ter apresentado eficiência de controle superior aos demais tratamentos, não se observou variação na produtividade em relação à testemunha, o que concorda com Arleu et al. (1981) e Sanches (1978), que encontraram eficiência de controle do ácaro alaranjado em abacaxizeiro usando o Vamidothion em pulverização pós-plantio, mas não obtiveram efeitos sobre a produtividade. Provavelmente, o nível da população (intensidade de infestação) por planta não determinou níveis de intensidade de danos foliares capazes de causar perdas, em termos de redução de produtividade.

Tratando-se de uma praga com escassa literatura sobre métodos de controle, inclusive com poucos relatos sobre controle químico e especialmente sobre a relação ácaro/planta, as informações obtidas poderão auxiliar numa tomada de decisão, em áreas onde a infestação do ácaro estiver estabelecida e/ou sem manejo, através do uso racional de insetici- 
das-acaricidas como medida complementar de controle, mantendo-a desse modo, em baixos níveis de infestação, minimizando os danos e prováveis perdas provocadas pelo ácaro alaranjado do abacaxizeiro. Determina, também, a necessidade de realização de novas pesquisas, comparando do sagens, freqüência e número de aplicações de inseticidas-acaricidas, ou acaricidas específicos, sistêmicos ou não, medição de perdas e estudos ecológicos (níveis maiores de infestação) na relação ácaro/planta.

\section{CONCLUSÕES}

- O tratamento com o inseticida-acaricida sistêmico Vamidothion é eficiente no controle do D. floridanus, enquanto que o Acefato mostrouse ineficiente.

- A aplicação dos inseticidas-acaricidas sistêmicos no controle do ácaro não interferiu na produtividade da cultura do abacaxi.

\section{REFERÊNCIAS BIBLIOGRÁFICAS}

ABBOTT, W.S. 1925. A Method of Computing the Effectiveness of an Insectide. Jour. of Economic Entomology, Maryland, v.18, n.1, p.265-267.

ARLEU, J.R.; T.B. PISSARRA; J.M. MUNIZ, 1981. Controle Químico do Ácaro Dolichotetranychus floridanus (Banks, 1900) e Sua Influência na Produção de Frutos e na Ocorrência de Fusariose do Abacaxizeiro. Cariocica: EMCAPA. 14p. (Boletim Técnico, 8). ARRUDA, G.P.; E.H.W. FLECHTMANN. 1967. Murcha de Abacaxizeiro Causado por Ácaro. Rev. Agricultura. Piracicaba, v.42, n.4, p. 172 .

CHALFOUN, S.M. 1998. Abacaxicultura Brasileira e o Mercado Globalizado. Informe Agropecuário, Belo Horizonte, v.19, n. 195, p.5-6. 
CHOAIRY, S.A. 1985. A Cultura do Abacaxi: Prática de Cultivo. João Pessoa: EMEPA. 21p. (Circular Técnica, 1).

CUNHA, G.A.P.; A.P.MATOS; J.R.S.CABRAL; L.F.S.SOUZA; N.F. SANCHES; D.H.R.C. REINHARDT. 1994. Abacaxi para Exportação: Aspectos Técnicos da Produção. Brasília: MAARA/ Embrapa. 41p. (Série Publicações Técnicas FRUPEX, 11).

HENDERSON, C.F.; E.W. TILTON. 1955. Test With Acaricides Against the Brown Wheat Mite. J. Econ: Entomol., v.63, n.6, p.1536-1539. MANICA, I. 1999. Fruticultura Tropical 5 - Abacaxi. Porto Alegre, Ed. Cinco Continentes, $581 \mathrm{p}$.

PIMENTEL-GOMES, F. 1990. Curso de Estatística Experimental. Piracicaba, 468p.

REIS, P.R. 1981. Pragas do Abacaxizeiro. Informe Agropecuário, Belo Horizonte, v.7, n.74, p.29-32.

SAMPAIO, R.S.; L. MYAZAKI. 1982. Ensaio Preliminar de Controle Químico ao Ácaro Plano do Abacaxizeiro. Pesq. Agropecuária Brasileira, Brasília, v.17, n.4, p.659-661.

SANCHES, N.F. 1978. Pragas do Abacaxi. Salvador: Embrapa-CNPMF. $12 \mathrm{p}$.

SANCHES, N.F.; A.C. ZEM. 1978. Ação de Diferentes Acaricidas no Controle do Ácaro Dolichotetranychus floridanus (Banks, 1900) em Mudas de Abacaxizeiro "PeroIa". In: CONGRESSO LATINO AMERICANO DE ENTOMOLOGIA, 3, 1978, Anais: Itabuna, SEB. 